\title{
A SIMPLIFIED PROOF OF A CONJECTURE OF D.G. KENDALL CONCERNING SHAPES OF RANDOM POLYGONS
}

\author{
IGOR N. KOVALENKO \\ National Academy of Sciences of Ukraine \\ V.M. Glushkov Institute of Cybernetics \\ 252027 Kyiv Ukraine ${ }^{1}$
}

(Received January, 1998; Revised January, 1999)

\begin{abstract}
Following investigations by Miles, the author has given a few proofs of a conjecture of D.G. Kendall concerning random polygons determined by the tessellation of a Euclidean plane by an homogeneous Poisson line process. This proof seems to be rather elementary. Consider a Poisson line process of intensity $\lambda$ on the plane $\Re^{2}$ determining the tessellation of the plane into convex random polygons. Denote by $K_{\omega}$ a random polygon containing the origin (so-called Crofton cell). If the area of $K_{\omega}$ is known to equal 1 , then the probability of the event \{the contour of $K_{\omega}$ lies between two concentric circles with the ratio $1+\varepsilon$ of their ratio $\}$ tends to 1 as $\lambda \rightarrow \infty$.
\end{abstract}

Key words: Stochastic Geometry, Random Sets, Random Tessellation.

AMS subject classifications: $60 \mathrm{D} 05$.

\section{Introductory Remarks}

Investigations of Miles [5] are devoted to the solution of the following problem suggested by Professor D.G. Kendall during WWII, and exposed in his Foreword to Stoyan, Kendall and Mecke [6]. This is a Poisson line process on the Euclidean plane. This line process determines the tessellation of the plane into convex random polygons. D.G. Kendall conjectured that the shape of a random polygon is close to a disk given that the area $A$ of the polygon is large. This is equivalent to considering $A$ to be fixed (for example, $A=1$ ), and the intensity of the line process to be large. Miles [5] uses some advanced approaches to this problem, but his proof is heuristic in some respects.

In the paper [2], the asymptotic behavior of the distribution function of the area of a Crofton cell was investigated; the result was expressed in terms of eigenvalues.

\footnotetext{
${ }^{1}$ The paper was written at the STORM Research Center, University of North London.
} 
The author has given a few proofs of the conjecture of D.G. Kendall. All these proofs have some common ideas:

(i) A well-known inequality of Bonnesen [1] is used to majorate the probability of having a Crofton cell significantly deviated from a disk, by the probability of that the perimeter of such a Crofton cell is at least multiple $(1+\delta)$ of that of a disk of the same area.

(ii) Let $\mathscr{K}$ be a set of possible realizations of a random polygon $K_{\omega}$. A mapping $\mathscr{G} \rightarrow \mathcal{L}$ is defined such that the image of $\mathscr{K}$ belongs to a set $\mathcal{L}$ of a rather simple structure. This enables us to derive a bound for the probability $P\left\{K_{\omega} \in \mathcal{K}_{6}\right\}$.

It was conceived that a reasonable mapping should be adjusted to the following measure of eccentricity of a polygon $K$ : if a polygon $K$ can be captured in a rectangle no smaller than $h \times H$ where $h$ is the width of $K$, then such a measure can be defined as $H / h(H>h)$.

In Kovalenko [3] it was suggested to map $K_{\omega}$ to the maximal inscribed lattice polygon, with the lattice cell size dependent on $m: 2^{m} \leq H / h<2^{m+1}$; moreover, the directions of the reference system axes were dependent on the $h \times H$ rectangle. In an unpublished paper, we simplified the approach, considering a square lattice with a given orientation, though the size of a lattice cell was chosen as $O(1 / m)$ for an $m$ such that $m^{2} \leq H / h<(m+1)^{2}$. Moreover, an inscribed lattice segment, a "needle", turned out to be sufficient for a proper bound rather than the maximal inscribed lattice polygon. In Kovalenko [4] the idea of approximating a random polygon by an inscribed polygon, with a bounded number of vertices included to the set of vertices of the former polygon, was proposed. In this paper, this idea is also used, but the approach is simplified to the extent such that the proof of the conjecture of D.G. Kendall can be regarded as a rather elementary proof.

\section{Main Results}

Let $\omega=\left\{\left(p_{n}, \varphi_{n}\right)\right\}$ be a two-dimensional Poisson point process with planar intensity $\lambda / \pi$ in the half-band $\mathscr{B}_{+} \times(0,2 \pi)$. For each point $\left(p_{n}, \varphi_{n}\right)$ of this point process, draw a line $l_{n}$ with polar coordinates $\left(p_{n}, \varphi_{n}\right)$. [This means that $\left(p_{n}, \varphi_{n}\right)$ is the point of $l_{n}$ closest to the origin.] We will also use the term "line $\left(p_{n}, \varphi_{n}\right)$ ". The set of lines $\left\{l_{n}\right\}$ is a Poissonian line process with intensity $\lambda$. This process determines the tessellation of the plane $\mathscr{R}^{2}$ into convex random polygons. A random polygon containing the origin is called a Crofton cell; it will be denoted by $K_{\omega}$. It is defined uniquely almost surely.

Let $A(K)$ be the area of a polygon $K$ and $r(K)$ be the minimal positive value $\rho$ such that the contour of $K$ can be enclosed between two concentric circles with the ratio $1+\rho$ of their radii. The function $r(K)$ can be considered as a measure of "noncircularity" of the polygon $K$. Our objective is to prove the following statement:

Theorem 1: Given an $\varepsilon>0$, the following relation holds true:

$$
P\left\{r\left(K_{\omega}\right)>\varepsilon \mid A<A\left(K_{\omega}\right)<A(1+h)\right\} \rightarrow 0 \text { as } \lambda \sqrt{A} \rightarrow \infty
$$

uniformly in $h$ varying in a sufficiently small interval $\left(0, h_{0}\right)$. 
Note: Let $\left\{\left(p_{n}, \varphi_{n}\right)\right\}$ be a Poissonian line process of intensity $\lambda$. Then $\left\{\left(c p_{n}, \varphi_{n}\right)\right\}$ is a process of the same kind, with intensity $\lambda / c$, for a positive $c$. A Crofton cell, as well as other random polygons, are transformed homothetically with dilation factor $c$. The above implies a dependence of the left-hand side of Equation (1) on $\varepsilon$ and $\lambda \sqrt{A}$ only. Without a loss of generality, $A$ can be set to 1 . Hence, Theorem 1 evidently follows from the following statements:

Theorem 2: Given a value $\beta>0$, the following bound holds true:

$$
P\left\{1<A\left(K_{\omega}\right)<1+h\right\}>\exp \{-2 \lambda(1+\beta) / \sqrt{\pi}\} h
$$

where $h>0$ is sufficiently small and $\lambda$ is sufficiently large.

Theorem 3: Given an $\varepsilon>0$, a value $\gamma>0$ can be chosen such that the following bound holds true:

$$
P\left\{1<A\left(K_{\omega}\right)<1+h, r\left(K_{\omega}\right)>\varepsilon\right\}<\exp \{-2 \lambda(1+\gamma) / \sqrt{\pi}\} h
$$

for sufficiently small $h>0$ and sufficiently large $\lambda$.

To derive Equation (1) from inequalities (2) and (3), it is sufficient to choose a $\beta$ with $0<\beta<\gamma$ and consider the ratio of inequalities (3) and (2).

\section{Proof of Theorem 2}

Consider a regular $N$-gon $K^{0}$ determined by lines $\left(p^{0}, \varphi_{i}^{0}\right)$ with $1 \leq i \leq N$, satisfying the following conditions:

(i) $\quad K^{0}$ is situated strictly inside the circle $C(O,(1+\beta) / \sqrt{\pi})$ where $C(O, R)$ hereinafter denotes the circle of the radius $R$, with the center at the origin; and

(ii) $\quad A\left(K^{0}\right)=1$.

[The latter is possible for sufficiently large $N$.]

One can choose a $\mu>0$ such that the property $(i)$ still holds true for a polygon $K$ determined by lines $\left(p_{i}, \varphi_{i}\right), 1 \leq i \leq N$, and, moreover, $K$ is an $N$-gon, as long as $\left|p_{i}-p^{0}\right| \leq \mu, \quad\left|\varphi_{i}-\varphi_{i}^{0}\right| \leq \mu, \quad 1 \leq i \leq N$. Denote $K \quad$ as $K_{x, y}$ where $x=$ $\left(p_{1}, \ldots, p_{N-1} ; \varphi_{1}, \ldots, \varphi_{N}\right)$ and $y=p_{N}$, and let $x^{0}=\left(p^{0}, \ldots, p^{0} ; \varphi_{1}^{0}, \ldots, \varphi_{N}^{0}\right)$. From a geometric argument, the area $A(x, y)$ of the polygon $K_{x, y}$ is a continuous function of $(x, y)$ as long as $\mu$ is small enough. One can observe that $A\left(x^{0}, p^{0}-\mu\right)<1$ and $A\left(x^{0}, p^{0}+\mu\right)>1$. Due to the continuity of the function $A(x, y)$ we have $A\left(x, p^{0}-\right.$ $\mu)<1$ and $A\left(x, p^{0}+\mu\right)>1+\sigma$ for $x \in G$, a neighborhood of $x^{0}$, and $\sigma$ being a positive constant. We have:

$$
0<\partial A(x, y) / \partial y<c_{0}:=2(1+\beta) / \sqrt{\pi}
$$

as $x \in G,\left|y-y^{0}\right| \leq \mu$, since this derivative is just the length of a side of $K_{x, y}$. By a continuity argument, values $y^{\prime}=y^{\prime}(x)$ and $y^{\prime \prime}=y^{\prime \prime}(x)$ can be chosen such that $A\left(x, y^{\prime}\right)=1$ and $A\left(x, y^{\prime \prime}\right)=1+h$, provided that $x \in G$ and $h<\sigma$. The Lagrange Theorem yields the bound $y^{\prime \prime}-y^{\prime}>h / c_{0}$, due to inequality (4).

Consider the following event for the point process $\left\{\left(p_{n}, \varphi_{n}\right)\right\}$ :

(i) for $N$ points $\left(p_{1}, \varphi_{1}\right), \ldots,\left(p_{N}, \varphi_{N}\right)$ the relations $X \in G, y^{\prime}(X)<Y<y^{\prime \prime}(X)$ hold, where random variables $X$ and $Y$ are defined as follows: 


$$
X=\left(p_{1}, \ldots, p_{N=1} ; \varphi_{1}, \ldots, \varphi_{N}\right), Y=p_{N}, \text { and }
$$

(ii) $\quad p_{n}>(1+\beta) / \sqrt{\pi}$ for all the rest $n$.

Evidently, conditions $(i)$ and $(i i)$ imply the bound $1<A\left(K_{\omega}\right)<1+h$. The probability of the defined event exceeds the value $(\lambda / \pi)^{N}|G| h \times e^{-2 \lambda(1+\beta) / \sqrt{\pi}} / c_{0}$ which, in its turn, exceeds the value $e^{-2 \lambda(1+\beta) / \sqrt{\pi}} h$ for sufficiently large $\lambda$, where

$$
|G|=\int_{\dddot{G}} \int d p_{1} \ldots d p_{N-1} d \varphi_{1} \ldots d \varphi_{N} .
$$

\section{Some Constructions with Convex Polygons}

Instead of the event $\left\{1<A\left(K_{\omega}\right)<1+h\right\}$, we consider first the event $\left\{1<A\left(K_{\omega}\right)<\right.$ $2\}$ in our upper bounds. Thus it is convenient to consider a class $\mathscr{K}$ of (nonrandom) convex polygons $K$ such that:

(i) $O \in K$

(ii) $\quad 1<A(K)<2$;

(iii) $\quad r(K)>\varepsilon$, where $\varepsilon$ is a fixed positive number.

Let $h(K)$ be the width of a convex polygon $K$. [Due to a common definition, the width means the minimal distance between two parallel lines surrounding $K$.] Also let $H(K)$ be the minimal value $H$ for which the $K$ can be contained in an $h(K)$ by $H$ rectangle. Evidently, $h(K) \leq H(K)$. Also denote the perimeter of a polygon $K$ by $S(K)$, and introduce classes $\overline{\mathscr{K}}_{m}=\mathscr{K} \cap\left\{K: m^{2} \leq H(K) / h(K)<(m+1)^{2}\right\}$.

Then

$$
q=\sum_{m \geq 1} q_{m}
$$

where

$$
q=P\left\{K_{\omega} \in \mathscr{K}_{\mathcal{G}}\right\}, q_{m}=P\left\{K_{\omega} \in \mathscr{K}_{m}\right\}
$$

Lemma 1: Every polygon $K \in \mathfrak{K}_{m}$ is situated inside the disk $C(O, 8 m)$. Moreover, $2 m<S(K)<16 m$.

Proof: Let $K$ be a polygon, $K \in \mathscr{K}_{m}$. Denote, for simplicity, $A(K)=A, H(K)=$ $H, h(K)=h, S(K)=S$. Evidently, $1<A<H h$. Hence, $1<\left(H / m^{2}\right) H=H^{2} / m^{2}$; $H>m$; thus $S>2 m$.

By our assumption, $K$ can be inscribed in an $h \times H$ rectangle and it includes points incident to each side of the rectangle; points $w_{1}$ and $w_{2}$ belong to $h$-sides, whereas $w_{3}$ and $w_{4}$ belong to $H$-sides; moreover, the segment $\left[w_{3}, w_{4}\right]$ can be assumed being parallel to $h$-sides. The triangles $w_{1} w_{3} w_{4}$, and $w_{2} \quad w_{3} w_{4}$ are contained by $K$. They have the common base $\left[w_{3}, w_{4}\right]$ and altitudes $H_{1}$ and $H_{2}$ such that $H_{1}+H_{2}=$ $H$. Hence, $2>A \geq H h / 2>H^{2} /\left(2(m+1)^{2}\right)$ and, consequently, $H<2(m+1)$. Since $O \in K$, one can observe that $K$ is situated inside the circle of radius $2 H<4(m+$ 1) $\leq 8 \mathrm{~m}$. Hence, $S \leq 4 H<16 \mathrm{~m}$.

Lemma 2: The inequality

$$
q_{m}<2^{14}(m \lambda)^{4} \exp \{-2 m \lambda / \pi\}
$$


holds whenever $\lambda>1 / 4$.

Proof: Set $h=h\left(K_{\omega}\right), H=H\left(K_{\omega}\right)$. Consider points $w_{1}, w_{2}$ to be included in $K_{\omega}$ and adjacent to opposite $h$-sides of the $h \times H$ rectangle including $K_{\omega}$.

Almost surely, only two events can occur:

(i) $\quad\left[w_{1}, w_{2}\right]$ is a side of $K_{\omega} ;$ and

(ii) it is a diagonal of $K_{\omega}$.

In case $(i)$, the following event certainly occurs. Three random lines $\left(p_{k}, \varphi_{k}\right)$, $k=1,2,3$ exist such that the first line is crossed by the other two inside the circle $C(O, 8 m)$; moreover, the segment between the two crossings is longer than $m$ and it is not crossed by an extra random line. The probability of the event $(i)$ is less than the value

$$
(1 / 2)(2 \lambda \times 8 m)^{3} e^{-2 m \lambda / \pi}=2^{11}(m \lambda)^{3} e^{-2 m \lambda / \pi} .
$$

In case $(i i)$ four random lines must exist such that no extra random line crosses the segment between the intersections of the first and second lines and the third and fourth lines. The conditions on the location of the four random lines and the distance between the two intersections are the same as in case $(i)$. As the result, we have the bound

$$
(3 / 4 !)(2 \lambda \times 8 m)^{4} e^{-2 m \lambda / \pi}=2^{13}(m \lambda)^{4} e^{-2 m \lambda / \pi}
$$

for the case (ii). The sum of bounds (8) and (9) yields bound (7).

\section{Two Geometric Lemmas}

Lemma 3: Given $\varepsilon>0$, a value $\delta>0$ can be chosen such that the inequalities $A(K)>1$ and $r(K)>\varepsilon$ for any closed convex figure $K$ imply the bound

$$
S(K)>2 \sqrt{\pi}(1+\delta) .
$$

Proof: Let $A, S, R$, and $r$ denote the area, perimeter, and outer and inner radii of a convex figure $K$, respectively. Recall the well-known inequality of Bonnesen [1]:

$$
S^{2}-4 \pi A \geq \pi^{2}(R-r)^{2} .
$$

Inequality (11) implies that if inequality (10) is not satisfied, then $R-r<\sqrt{\delta^{\prime} / \pi}$ where $\delta^{\prime}=2 \delta+\delta^{2}$. Assume that $\delta^{\prime}<1 / 16$. By the definition of outer and inner radii, $C_{r} \subset K \subset C_{R}$, where $C_{r}, C_{R}$ are disks of the radii $r, R$ respectively. A disk $C_{\rho}$ of radius $\rho=2 r-R$ can be formed such that $C_{\rho} \subset C_{r}, C_{\rho}$ is concentric to $C_{R}$. [Since $\quad R>1 \sqrt{\pi}$, we have $2 r-R=R-2(R-r)>\left(1-r \sqrt{\delta^{\prime}}\right) / \sqrt{\pi}>0$.] Furthermore:

$$
R / \rho<R /\left(R-4 \sqrt{\delta^{\prime} / \pi}\right)<1 /\left(1-4 \sqrt{\delta^{\prime}}\right):=1+\varepsilon .
$$

Therefore, $r(K) \leq \varepsilon$. Considering the logically opposite events, one observes that $\{A(K)>1, r(K)>\varepsilon\} \Rightarrow\{S(K)>2 \sqrt{\pi}(1+\delta)\}$, where $\varepsilon$ and $\delta$ are related according to the Equation (12). 
Lemma 4: Given $\gamma^{\prime}>0$, a number $\nu$ can be chosen such that the following property holds. For an arbitrary convex polygon $K$, another convex polygon $L$ exists such that:

(i) L is at most $\nu$-gon;

(ii) each vertex of $L$ is a vertex of $K$; and

(iii) $S(L)>\left(1-\gamma^{\prime}\right) S(K)$.

Proof: Denote vertices of $K$ by $\left(p_{i}^{\prime}, \varphi_{i}^{\prime}\right)$, where $1 \leq i \leq N, 0<\varphi_{1}^{\prime}<\ldots<\varphi_{N}^{\prime}<2 \pi$.

Set $i(k)=\arg \max \left\{p_{i}^{\prime} \cos \left(\varphi_{i}^{\prime}-2 \pi k / \nu\right)\right\}, 0<k<\nu-1$, where $\nu \geq 3$, and define $L$ as the convex hull of points $\left(p_{i(k)}^{\prime}, \varphi_{i(k)}^{\prime}\right)$.

Evidently, $L$ is at most $\nu$-gon. It can be observed that $K \backslash L$ is a union of convex polygons $D_{j}$ possessing the following properties:

(i) The contour of each $D_{j}$ consists of side $S_{j}$ of polygon $L$, and some sides $S_{j l}$ of the polygon $K$;

(ii) $\quad D_{j}$ is captured in a triangle $\nu_{1} \nu_{2} \nu_{3}$, say, where $\left[\nu_{1} \nu_{2}\right]=S_{j}$ and the angle opposite to it equals $\pi-2 \pi / \nu$.

It can be easily shown that

$$
\left|S_{j}\right| \geq\left(\left|\nu_{1} \nu_{3}\right|+\left|\nu_{2} \nu_{3}\right|\right) \cos (\pi / \nu) \geq \sum_{l}\left|S_{j l}\right| \cos (\pi / \nu)
$$

Summing up the above inequalities in $j$, we arrive at the inequality

$$
S(L) \geq S(K) \cos (\pi / \nu)
$$

Therefore, the lemma statement holds true given that number $\nu$ satisfies the inequality $\cos (\pi / \nu)>1-\gamma^{\prime}$.

\section{A Bound for $q_{1}$}

Assume that $K \in \mathscr{K}_{1}$. By Lemmas 3 and 4 ,

$$
S(L)>S(K)\left(1-\gamma^{\prime}\right)>2 \sqrt{\pi}(1+\delta)\left(1-\gamma^{\prime}\right) .
$$

The number $j$ of sides of $K$ adjacent to vertices of $L$ is at most $2 \nu$. Given these $j$ lines, the polygon $L$ can be chosen in at most $2^{j+1}$ ways. [Indeed, two events can occur:

(i) none of these lines contains a side of $L$,

(ii) there are some.

In case $(i)$, there are two ways of coupling the lines to obtain vertices of $L$. In case (ii), it is sufficient to choose a non-empty subset of lines: as those containing the sides of $L$. There are $2+\left(2^{j}-1\right)<2^{j+1}$ ways altogether]. If $L$ is chosen, then the probability of no crossing of $L$ by an extra random line equals $e^{-\lambda S(L) / \pi}$. Finally, by Lemma $1, p_{i}<8$ for all $j$ random lines. As a result, a bound can be obtained as follows:

$$
q_{1}<2 \sum_{j=3}^{2 \nu} \frac{(32 \lambda)^{j}}{j !} \exp \left\{-2 \lambda(1+\delta)\left(1-\gamma^{\prime}\right) / \sqrt{\pi}\right\}
$$


Choose $\gamma^{\prime}=8 \delta / 17$, where $\delta<1 / 16$. Then the bound

$$
q_{1}<e^{-(2+\delta) \lambda / \sqrt{\pi}}
$$

takes place for sufficiently large $\lambda$.

\section{Proof of Theorem 3}

It is sufficient to pass from integral to local bounds.

We have

$$
q_{m}=\sum_{n \geq 3} q_{m n}
$$

where $q_{m n}$ is the contribution of $n$-gons to $q_{m}$. In turn,

$$
q_{m n}=\int_{R} \ldots \int e^{-S \lambda / \pi} \prod_{i=1}^{n}(\lambda / \pi) d p_{i} d \varphi_{i}
$$

where $S$ is the perimeter and the domain $R=R_{m n}$ is defined by the condition that the lines $\left(p_{i}, \varphi_{i}\right)$ form a convex $n$-gon $K \in \mathscr{K}_{m}$. It is convenient to introduce a scale parameter $x=p_{1}$ and a "shape parameter" $z=\left(p_{2} / p_{1}, \ldots, p_{n} / p_{1} ; \varphi_{1}, \ldots, \varphi_{n}\right)$. Denote by $S(z, x)$ the perimeter of the $n$-gon coded as $(z, x)$ and by $a(z, x)$ the positive square root of its area. Equation (15) implies the formula

$$
q_{m n}=\int_{U} f(z) d z \int_{\xi<x<\xi \sqrt{2}} x^{n-1} \exp \{-\lambda S(z, x) / \pi\} d x,
$$

where $f(z) d z$ is an elementary probability, $\xi=\xi(z)$ is the root of the equation $a(z, \xi)=1$, and $U$ is a $2 n-1$-dimensional domain.

Evidently, the following similarity conditions hold true:

and

$$
a(z, x)=x / \xi
$$

$$
S(z, x)=s x / \xi
$$

with $s=S(z, \xi)$.

The right-hand side of Equation (16) is related to the event $\left\{1<A\left(K_{\omega}\right)<2\right\}$. As for event $\left\{1<A\left(K_{\omega}\right)<1+h\right\}$, we have a similar integral with $x$ varying in the interval $\xi<x<\xi \sqrt{1+h}<\xi(1+h / 2)$ instead of $\xi<x<\xi \sqrt{2}$; see Equation (17). We have

$$
\begin{gathered}
\int_{\xi}^{\xi+\xi h / 2} x^{n-1} e^{-\lambda s x /(\xi \pi)} d x=(\xi h / 2) \eta^{n-1} e^{-\lambda s \eta /(\xi \pi)} \\
<(h /(2 \pi)) \lambda s \int_{\xi}^{\xi \sqrt{2}} x^{n-1} e^{-\lambda s x /(\xi \pi)} d x /\left(1-e^{-\lambda s(\sqrt{2}-1-h / 2) / \pi}\right) .
\end{gathered}
$$


By Lemma 1, $2 m<s<16 m$; hence inequality (19) implies the bound

$$
\int_{\xi}^{\xi+\xi h / 2} x^{n-1} e^{-\lambda s x /(\xi \pi)}<(8 / \pi) h \lambda m \int_{\xi}^{\xi \sqrt{2}} x^{n-1} e^{-\lambda s x /(\xi \pi)} d x(1+o(1)) .
$$

Integrating both sides of Equation (20) in $z$, with a weight function $f(z)$ and summing up over $n>3$ one obtains the bound

$$
P\left\{K_{\omega} \in \mathscr{K}_{m}, 1<A\left(K_{1}\right)<1+h\right\}<(8 / \pi) h \lambda m q_{m}(1+o(1)) .
$$

From Lemma 2 and bound in Equation (13), we obtain the bound

$$
\begin{gathered}
P\left\{K_{\omega} \in \mathscr{G}, 1<A\left(K_{\omega}\right)<1+h\right\} \\
<(8 / \pi) h \lambda\left(e^{-(2+\delta) \lambda / \sqrt{\pi}}+2^{14} \lambda^{4} \sum_{m=2}^{\infty} m^{5} e^{-2 \lambda m / \pi}\right)(1+o(1))
\end{gathered}
$$

for sufficiently small $h$ and large $\lambda$. Having chosen a value $\gamma>0$, such that the inequality $\gamma<(\delta / 2) \wedge((2 / \sqrt{\pi})-1)$ is satisfied, one yields the statement of Theorem 3 .

\section{Densities for a Finite $\lambda$}

The derived Equation (1) expresses the essence of the conjecture of D.G. Kendall. Nevertheless, it would be also desirable to get a corresponding equation for densities:

$$
P\left\{r\left(K_{\omega}\right)>\varepsilon \mid A\left(K_{\omega}\right)=A\right\} \rightarrow 0 \text { as } \lambda \sqrt{A} \rightarrow \infty .
$$

Evidently, the existence of densities

$$
\lim (1 / A h) P\left\{A<A\left(K_{\omega}\right)<A(1+h)\right\} \text { as } h \rightarrow 0
$$

and

$$
\lim (1 / A h) P\left\{r\left(K_{\omega}\right)>\varepsilon, A<A\left(K_{\omega}\right)<A(1+h)\right\} \text { as } h \rightarrow 0
$$

would be sufficient for deriving Equation (22) from Theorem 1, as Equation (1) means the existence of the double limit of the considered ratio as $h \rightarrow 0$ and $\lambda \sqrt{A} \rightarrow \infty$. As limits (23) and (24) are assumed to exist, one may set $h \rightarrow 0$ and then $\lambda \sqrt{A} \rightarrow \infty$; as the result, one obtains Equation (22).

To avoid trivial cumbersomeness, we will investigate the existence of the limit

$$
\lim (1 / h) P\left\{\left(\text { shape of } K_{\omega}\right) \in L, 1<a\left(K_{\omega}\right)<1+h\right\} \text { as } h \rightarrow 0
$$

for every $\lambda>0$ assuming that $L=\sum_{m \geq 1} \sum_{n \geq 3} L_{m n}$, where each $L_{m n}$ is an arbitrary Borel set of shapes $z=\left(z_{1}, \ldots, z_{n-1} ; \varphi_{1}, \ldots, \varphi_{n}\right)$ relating to the $n$-gons with 
$m^{2} \leq H(K) / h(K)<(m+1)^{2} ; a(K)=A^{1 / 2}(K)$.

To prove the existence of the conditional probability $P\left\{r\left(K_{\omega}\right)>\varepsilon \mid a\left(K_{\omega}\right)=1\right\}$, it is sufficient to choose two versions of $L_{m n}$ :

(i) $\quad\left\{K\right.$ is an $n$-gon: $\left.r(K)>\varepsilon ; m^{2} \leq m(K) / h(K)<(m+1)^{2}\right\}$ and

(ii) $\quad\left\{K\right.$ is an $n$-gon: $\left.\left.m^{2} \leq H(K) / \overline{h(} K\right)<(m+1)^{2}\right\}$.

The transition from the condition $\left\{a\left(K_{\omega}\right)=1\right\}$ to the condition $\left\{a\left(K_{\omega}\right)=a\right\}$ is trivial, due to the similarity property mentioned just after the formulation of Theorem 1. Finally, the transformation $A\left(K_{\omega}\right)=a^{2}\left(K_{\omega}\right)$ yields the existence of corresponding densities for $A\left(K_{\omega}\right)$.

Therefore, it would be sufficient to establish the existence of limit (25). Consider $\lambda$ as a positive constant. Analogously to Equation (15), we arrive at relation

$$
\begin{gathered}
\Delta_{m n}(h):=(1 / h) P\left\{\left(\text { shape of } K_{\omega}\right) \in L_{m n}, 1<a\left(K_{\omega}\right)<1+h\right\} \\
=\int \ldots \int(\lambda s(z) / \pi)^{n} d z \int_{L_{m n}}^{1+h} \frac{1}{h} x^{n-1} e^{-\lambda s(z) x / \pi} d x .
\end{gathered}
$$

Similar to Equation (19), taking also into account that $2 \sqrt{\pi}<s<16 m$, we obtain

$$
\begin{array}{r}
\frac{1}{h} \int_{1}^{1+h} x^{n-1} e^{-\lambda s x / \pi} d x \\
<c m \int_{1}^{\sqrt{2}} x^{n-1} e^{-\lambda s x / \pi} d x
\end{array}
$$

for a constant $c$, and, as the limit, the inequality

$$
e^{-\lambda s / \pi}<c m \int_{1}^{\sqrt{2}} x^{n-1} e^{-\lambda s x / \pi} d x .
$$

Integrating inequalities (27) and (28) with weight function $(\lambda s(z) / \pi)^{n}$ over the region $L_{m n}$, we have

and

$$
\Delta_{m n}(h)<c m q_{m n}
$$

where

$$
\Delta_{m n}(0)<c m q_{m n}
$$

$$
\Delta_{m n}(0):=\int \underset{L_{m n}}{\ldots}(\lambda s(z) / \pi)^{n} e^{-\lambda s(z) / \pi} d z .
$$

Hence, due to bound (7),

(i) the series $\Delta(h):=\sum_{m, n} \Delta_{m n}(h)$ is uniformly convergent in the interval $0<h<h_{0}$ for positive $h_{0}$, 
(ii) the series $\Delta(0):=\sum_{m, n} \Delta_{m n}(0)$ is convergent,

(iii) $\quad \Delta_{m n}(h) \rightarrow \Delta_{m n}(0)$ as $h \rightarrow 0$.

Statements $(i),(i i)$ and $(i i i)$ imply the existence of the right-hand derivative of function $P\left\{\left(\right.\right.$ shape of $\left.\left.K_{\omega}\right) \in L, a\left(K_{\omega}\right)<x\right\}$ at the point $x=1$. The existence of the left-hand side derivative can be proved in a quite similar manner. Furthermore, it is easy to verify that the both are identical. As the consequence of the above, equation (22) holds true.

\section{Acknowledgements}

I am very grateful to Professor David G. Kendall for encouraging my studies of this problem and giving me helpful advises.

My deep gratitude goes to Professor R. Gilchrist who supported the elaboration of this paper in STORM and gave a lot of suggestions.

I had some discussions with other mathematicians and also reported my results in London Probability Seminar, Statistical Seminar in the University of Cambridge, Seminars in the University of Jena and Bergakademie Freiberg, Workshop on Random Sets in Edinburgh and others. All these discussions inspired me to elaborate a rather simple version of the proof of the Conjecture of D.G. Kendall. I am very much thankful for all who took part in the discussions.

Suggestions of the Principal Editor Jewgeni H. Dshalalow and Editor John Branard were very helpful in editing the text. I am greatful to both of them.

\section{References}

[1] Bonnesen, T., Über eine Vershärfung der isoperimetrischen Ungleichheit des Kreises in der Ebene und auf der Kugeloberflache nebst einer Anwendung auf eine Minkowskische Ungleichheit für konvexe Körper, Math. Ann. 84 (1921), 216-227.

[2] Goldman, A., Sur une conjecture de D.G. Kendall concernant la cellule de Crofton du plan et sur sa contrepartie brownienne, Compt. Rend. Acad. Sci. Paris 326:1 (1998), 233-237.

[3] Kovalenko, I.N., An alternative approach to R.E. Miles' proof of a conjecture of D.G. Kendall concerning the shapes of random polygons, Doc. No. 62810, STORM, University of North London (1997).

[4] Kovalenko, I.N., Proof of David Kendall's conjecture concerning the shape of large random polygons, Cybern. and Sys. Anal. 33:4 (1997), 461-467.

[5] Miles, R.E., A heuristic proof a long-standing conjecture of D.G. Kendall concerning the shapes of certain large random polygons, Adv. Appl. Prob. (SGSA) 27 (1997), 397-471.

[6] Stoyan, D., Kendall, W.S., Mecke, J., Stochastic Geometry and Its Applications, With a Forward by D.G. Kendall, John Wiley and Sons, New York 1987. 


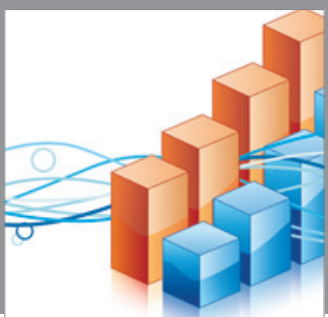

Advances in

Operations Research

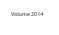

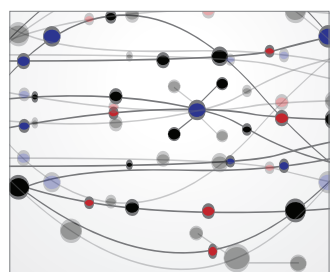

\section{The Scientific} World Journal
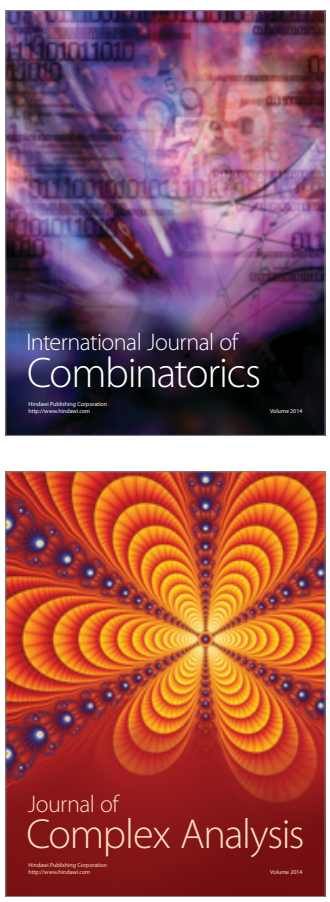

International Journal of

Mathematics and

Mathematical

Sciences
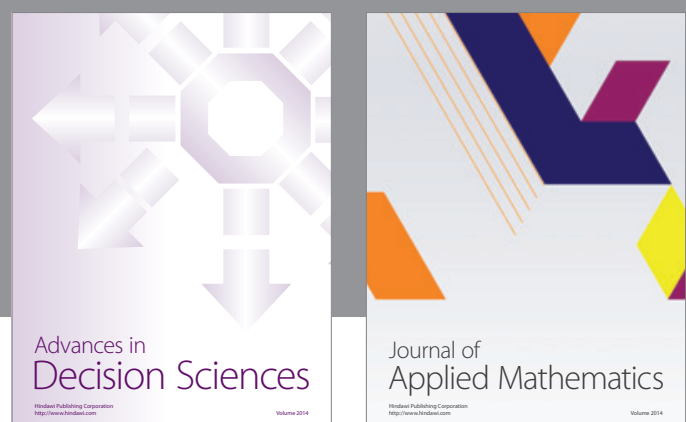

Journal of

Applied Mathematics
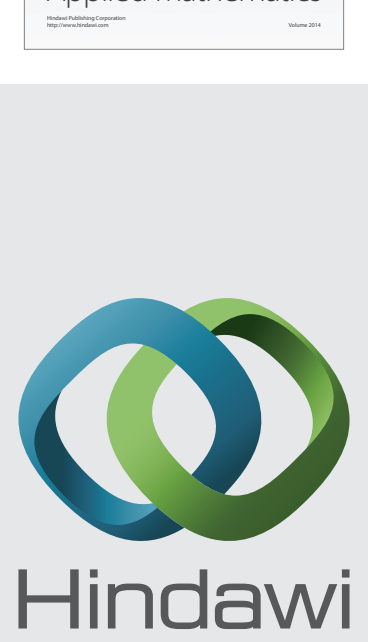

Submit your manuscripts at http://www.hindawi.com
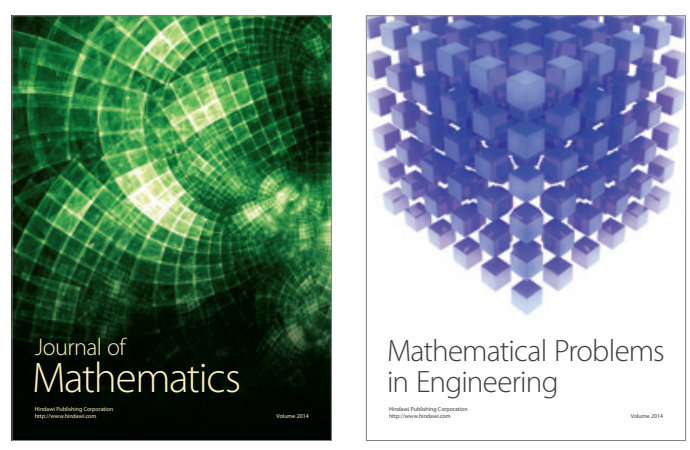

Mathematical Problems in Engineering
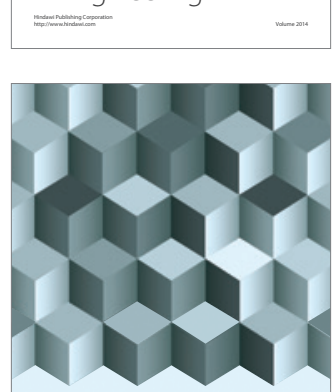

Journal of

Function Spaces
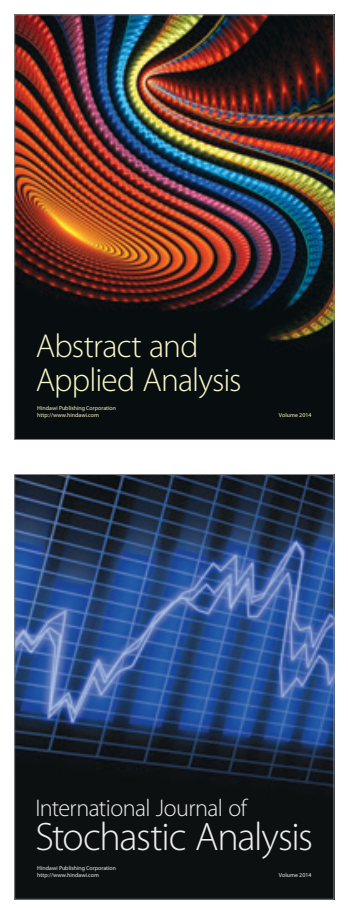

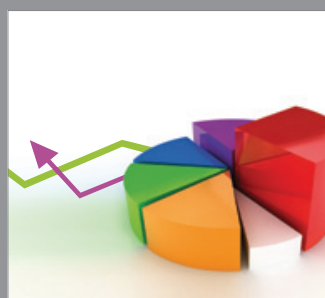

ournal of

Probability and Statistics

Promensencen
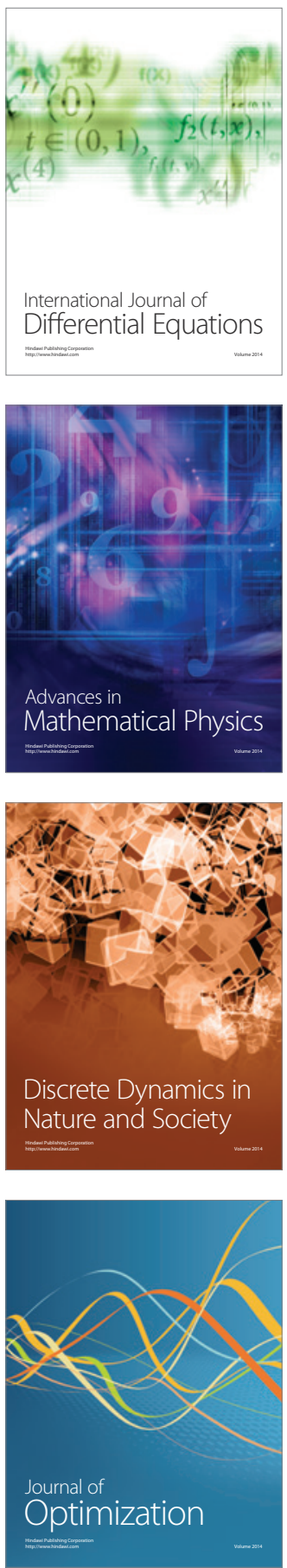